\title{
Limiting Solar Cell Heat-Up by Quantizing High Energy Carriers
}

\author{
L. Devarakonda and S. Mil'shtein \\ Department of Electrical and Computer Engineering, Advanced Electronic Technology Center, \\ 1 University Avenue, Lowell, MA 01854, USA \\ Correspondence should be addressed to S. Mil’shtein, samson_milshtein@uml.edu
}

Received 25 April 2012; Accepted 3 June 2012

Academic Editors: O. O. Fasina and M. Zoulias

Copyright ( $) 2012$ L. Devarakonda and S. Mil'shtein. This is an open access article distributed under the Creative Commons Attribution License, which permits unrestricted use, distribution, and reproduction in any medium, provided the original work is properly cited.

\begin{abstract}
Under solar radiation the efficiency of solar cells decreases as a result of heating up by short wavelengths photons. To minimize loss of efficiency with increasing temperatures, we designed a heterostructure AlGaAs/AlGaAs/GaAs cascaded p-i-n solar cells with 30 $\AA ̊$ wide quantum wells and $10 \AA$ wide barriers in p and i regions. Our modeling demonstrated that quantizing high energy carriers in the superlattice prevents scattering of excessive electron energies, thus decreasing the temperature rise per unit of time by a factor of 2. The modeling based on continuity equations included integration of absorption coefficients for various wavelengths and summarizes all thermodynamic heat exchanges in the designed solar cell.
\end{abstract}

\section{Introduction}

Effect of temperature on photovoltaic conversion has been a cause of much study for a long time. Rise in temperature does not significantly affect the light-generated current of the cell [1]. However, reverse saturation current increases exponentially with temperature. Furthermore, open circuit voltage reduces with temperature. It has been experimentally observed that the efficiency of a GaAs cell at $50^{\circ} \mathrm{C}$ drops by $10 \%$ from its efficiency at $28^{\circ} \mathrm{C}$ (i.e., $\eta_{50}=0.9 \eta_{28}$ ) [1]. It has been observed that the temperature of a c-Si cell array can reach up to about $55^{\circ} \mathrm{C}$ in about 25 minutes when exposed to a radiation of $1100 \mathrm{~W} / \mathrm{m}^{2}$ [2]. It is imperative to reduce the heat build-up in the cell in order to improve conversion efficiencies.

It was established that the reason for heat build-up during cell operation is the generation of hot electrons [3]. Incident solar radiation is a combination of light of various wavelengths or photons of various energies. A semiconductor of band gap $E_{g}$ would absorb photons of energy equal to or higher than the band gap. Energy in excess of the band gap is utilized in exciting electrons and holes to higher energy levels. These carriers (hot carriers) eventually relax back to the band edges, thereby liberating the excess energy as heat. For this reason, higher band gap materials are said to generate less heat. Successful usage of various energy gaps was demonstrated in multijunction cascade solar cell structure with tunnel junction [4] and, more recently, in design of AlGaAs/GaAs cascade structure [5]. It has been proved that large band gaps improve the cell efficiency at higher temperature due to smaller temperature coefficients for reverse saturation current (dark current). Structures involving quantum well, quantum dots, and quantum wires possess quantized energy level that can accommodate high energy electron, thereby providing larger effective band gaps. Experimental results proved expected temperature effects in p-i-n GaAs/AlGaAs QW solar cell for well periods of 23 and 40 in [6]. It has been observed that the cell with 40 QWs exhibits enhanced performance when compared to similar cells of well or barrier material.

In this study we demonstrate that performance of the solar cell can be improved by reducing the heat generated by hot carries. We use thermodynamic modeling in predicting the temperature rise in AlGaAs/AlGaAs/GaAs cascade p-i-n structure [5] under solar irradiance of AM1.5. We propose a model based on quantization and continuity equation to calculate photocurrent for a superlattice structure. We also show that use of superlattice structure would reduce the temperature rise in the cell. Section 1 elucidates the theoretical framework. The parameters of the $\mathrm{p}-\mathrm{i}-\mathrm{n}$ cascade 
cell are described in Section 2. In Section 3, we discuss the effect of QWs in $\mathrm{p}$ and $\mathrm{i}$ regions of the designed structure. Simulation results are presented in Section 4.

\section{Theory}

Consider a semiconductor material of band gap $E_{g}$. When this material is exposed to a photon of energy $h v>E_{g}$, carriers are excited to energy levels above band edges as in Figure 1. Eventually, these energetic carriers heat up the material though scattering mechanism. The heat energy that would be dissipated due to carrier relaxation following photon absorption is (it is assumed that all the excess energy is converted into thermal energy) given in (1):

$$
\Delta E=\Delta E_{e}+\Delta E_{h}=h v-E_{g} \mathrm{eV} .
$$

If $\Delta \Phi_{\lambda}$ is the photon flux $\left(\# / \mathrm{m}^{2} \mathrm{~s}\right)$ absorbed by the material, then the total power (density) dissipated as heat due to carrier scattering is given by (2):

$$
P_{H \lambda}=\left(\Delta E_{e}+\Delta E_{h}\right) \Delta \Phi_{\lambda}=\Delta E \Phi_{0 \lambda}\left(1-e^{-\alpha_{\lambda} W}\right) \frac{W}{m^{2}},
$$

where $\Phi_{0 \lambda}$-photon flux (at wavelength $\lambda$ ) at the surface, $W$ thickness, $\alpha_{\lambda}$-absorption coefficient.

Thus, power dissipated in heat due to carrier scattering in a heterostructute (multilayer) structure is given in (3):

$$
P_{H \lambda}=\sum_{k}\left(\Delta E_{e k}+\Delta E_{h k}\right) \phi_{0 \lambda} e^{-\sum_{m}^{k-1} \alpha_{m \lambda} W_{m}}\left(1-e^{-\alpha_{k \lambda} W_{k}}\right) \frac{W}{m^{2}},
$$

where $\Delta E_{e k}, \Delta E_{h k}$-Electron energy above conduction band and hole energy above valance band in $k$ th layer, respectively, $W_{k}$-thickness of $k$ th layer, $\alpha_{k \lambda}$-absorption coefficient of $k$ th layer.

This heat is utilized in increasing the temperature of the materials. From basic laws of thermodynamics, the total thermodynamic power density required to raise the temperature of a multijunction cell though $\Delta T$ is given in (4):

$$
P_{H}=\left(\frac{\Delta T}{\Delta t}\right) \sum_{k=1}^{n} \rho_{k} W_{k} C_{k}
$$

where $m_{k}, \rho_{k}, C_{k}$-mass, density and specific heat of material in $k$ th layer. From (3) and (4), the rate of temperature rise is given by (5):

$$
\frac{\Delta T}{\Delta t}=\frac{P_{H \lambda}}{\sum_{k=1}^{n} \rho_{k} W_{k} C_{k}} .
$$

The photon flux at any given wavelength can be calculated from the solar spectrum data. In the present case, solar spectrum data for AM1.5 was utilized.

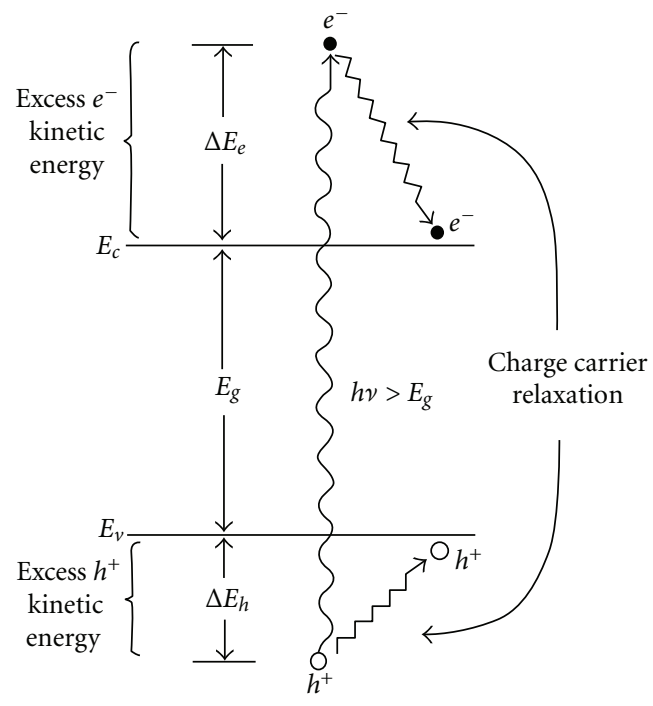

Figure 1: Hot carrier relaxation [3].

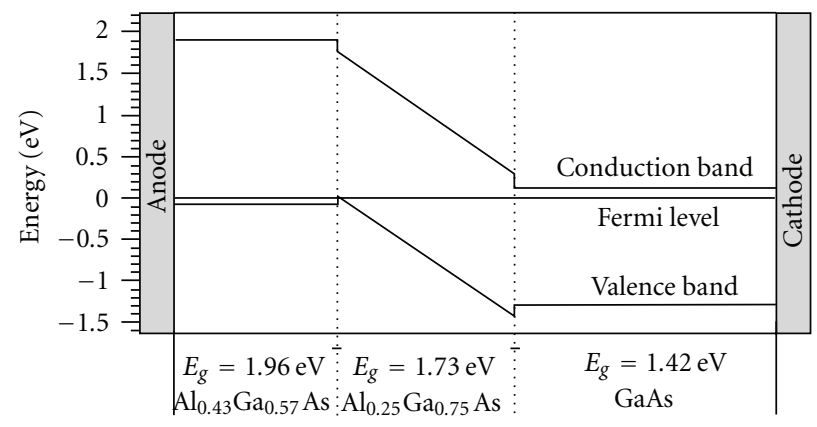

Figure 2: Band structure of $\mathrm{p}-\mathrm{i}-\mathrm{n}$ solar cell.

\section{Solar Cell Design}

3.1. I-V Characteristics. We designed a pin cascade solar cell with and without superlattices in $\mathrm{p}$ and $\mathrm{i}$ regions to address heating issues. First we consider the solar cell without superlattices (Figure 2). The materials for $\mathrm{p}-\mathrm{i}-\mathrm{n}$ regions were $\mathrm{Al}_{0.43} \mathrm{Ga}_{0.57} \mathrm{As} / \mathrm{Al}_{0.25} \mathrm{Ga}_{0.75} \mathrm{As} / \mathrm{GaAs}$, respectively. The $\mathrm{p}$-i-n thicknesses were $0.1 \mu \mathrm{m}, 4 \mu \mathrm{m}$, and $2 \mu \mathrm{m}$, respectively. The doping in $\mathrm{p}$ and $\mathrm{n}$ regions was $10^{18} / \mathrm{cm}^{3}$.

The dark current for such a cell would be the reverse saturation diode current. Forward currents in p, i, and n regions were calculated by solving the continuity equation in each region [7]. The total current in each region is created by photogeneration of wavelengths above the band gap and is defined by (6):

$$
\begin{aligned}
J=\int_{0}^{\lambda} J_{\lambda} d \lambda= & \int_{0}^{1.24 / E_{g p}} J_{p}(\lambda) d \lambda \\
& +\int_{0}^{1.24 / E_{g i}} J_{i}(\lambda) d \lambda+\int_{0}^{1.24 / E_{g n}} J_{n}(\lambda) d \lambda,
\end{aligned}
$$

where $J$-total current density, $J_{\mathrm{p}, \mathrm{i}, \mathrm{n}}$-Current contribution from $\mathrm{p}, \mathrm{i}$, and $\mathrm{n}$ regions at a wavelength $\lambda$. This gives a current 


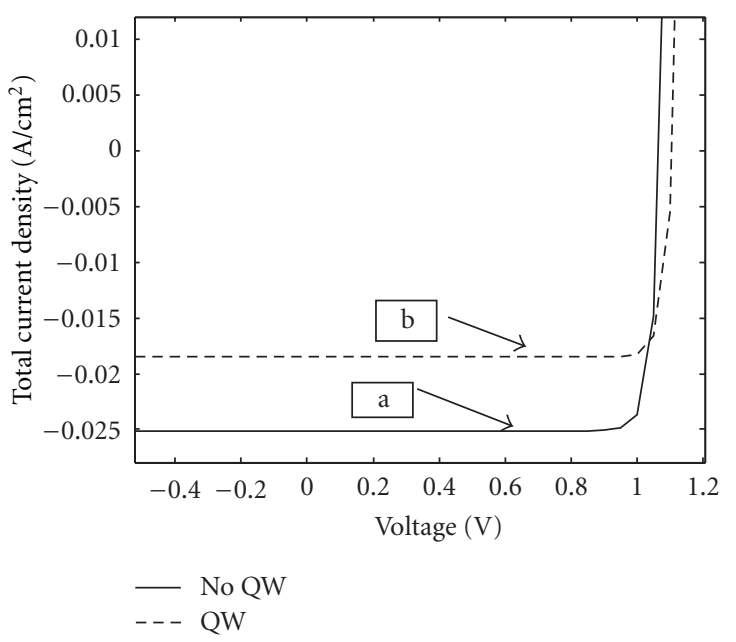

Figure 3: $I-V$ characteristics of a PIN cascade solar cell (a) without QW (b) with QWs.

density of $24.6 \mathrm{~mA} / \mathrm{cm}^{2}$. With the open circuit voltage of $1.04 \mathrm{~V}$ the efficiency was $25 \% . I-V$ characteristics are given in Figure 3.

3.2. Thermal Calculation. The power dissipated in heat is given by (5) for subindex $k=\mathrm{p}, \mathrm{i}, \mathrm{n}$.

\section{Controlling High Energy Carriers}

One-dimensional quantization of carriers can be achieved by superlattice built between high band gap and low band gap materials. The relative location of discrete energy levels from valance and conduction band edges was obtained by solving the Schrödinger wave equation for particles bounded by finite potential barriers. Finite difference method was used to convert the wave equation to a Hamiltonian matrix form. Eigenvalues of this matrix represent the energy levels, and the eigenvectors identify the wave function. The effective band gap of the region with quantum well would then be $E_{g}+\Delta E_{c n}+E_{v n}$, where $\Delta E_{c n}$ and $\Delta E_{v n}$ are $n$th energy level inside the well from conduction and valance band edges, respectively. These energy distances would define the energy of the photon that can be absorbed in the well.

Quantum well dimensions in a superlattice were selected to provide sufficiently high energy levels for carriers. The barrier thickness of about $10 \AA$ was chosen to ensure good quantum transparency. Aluminum phosphide (AlP) with a band gap of $3.57 \mathrm{eV}$ was used as a barrier material in $\mathrm{p}$ and i regions. This resulted in barriers of potential $0.954 \mathrm{eV}$ in conduction band and $0.636 \mathrm{eV}$ in valance band, respectively. In $\mathrm{i}$ region, this was $1.08 \mathrm{eV}$ in conduction band and $0.72 \mathrm{eV}$ in valance band. A well width of $30 \AA$ with $10 \AA$ barriers resulted in 4 energy levels in conduction and valance bands in $\mathrm{p}$ and i regions. Well period was 23 and 998, respectively, in $\mathrm{p}$ and $\mathrm{i}$ regions. Due to narrow barrier thickness, these energy levels penetrate into adjoining well. Since all the wells were identical, the energy levels were identical, resulting in resonant tunneling through the region. We feel that QWs in n-region might not be required as most of the high energy photon absorption takes place in $\mathrm{p}$ and i regions.

4.1. Carrier Transport through Superlattices Structure. Carrier transport can be analyzed by using a particle through a potential barrier paradigm $[8,9]$. Transmission and reflection for an electron at an energy level $\Delta E_{k}$ from a conduction band or valance band edges are related by (7):

$$
\left[\begin{array}{c}
T_{n} \\
0
\end{array}\right]=M^{n}\left[\begin{array}{c}
1 \\
R_{n}
\end{array}\right]=\left[\begin{array}{ll}
m_{11} & m_{12} \\
m_{21} & m_{22}
\end{array}\right]\left[\begin{array}{l}
1 \\
R
\end{array}\right],
$$

where

$$
\begin{aligned}
M=\frac{1}{4}\left[\begin{array}{cc}
e^{-i k_{w} L_{b}} & e^{-i k_{w} L_{b}} \\
e^{i k_{w} L_{b}} & -e^{i k_{w} L_{b}}
\end{array}\right] \cdot\left[\begin{array}{cc}
e^{-k_{b} L_{b}} & e^{k_{b} L_{b}} \\
\frac{i k_{b}}{k_{w}} e^{-k_{b} L_{b}} & \frac{-i k_{b}}{k_{w}} e^{k_{b} L_{b}}
\end{array}\right] \\
\cdot\left[\begin{array}{cc}
1-\frac{i k_{w}}{k_{b}} & 1+\frac{i k_{w}}{k_{b}} \\
1+\frac{i k_{w}}{k_{b}} & 1-\frac{i k_{w}}{k_{b}}
\end{array}\right], \\
k_{w}=\frac{\sqrt{2 m^{*} \Delta E_{k}}}{\hbar}, \quad k_{b}=\frac{\sqrt{2 m^{*}\left(V_{0}-\Delta E_{k}\right)}}{\hbar},
\end{aligned}
$$

$V_{0}$-Barrier potential above band edge.

Tunneling probability through $n$-barriers is given by $P n=T_{n} * T_{n}$.

4.2. I-V Characteristics. The current in the QWSC was obtained by solving the continuity equation for carrier concentration in each well and barrier regions with boundary conditions $\delta n_{k}\left(x \leq x_{0 k}\right)=0$. Here, $\delta n_{k}$ is the carrier concentration in the $k$ th well or barrier. The current contribution from $k$ th well or barrier (Figure 4 ) is given by (10)

$$
J_{k}=q P_{n-k} D_{k} \frac{d \delta n_{k}}{d x},
$$

where $D_{k}$-Diffusion coefficient of the material, $P_{n-k}$-Probability of tunneling through $n-k$ barriers obtained from Section 3.1.

Our model also took into account the fact that there would be no generation in barrier region for photons energies less than the barrier band gap. The wave nature of the electron would enable them to tunnel through the barriers. The presence of high field in i-region eliminates recombination. The current contribution in each region (at wavelength $\lambda$ ) is the sum of current due to each well and barrier regions. The total current due to entire spectrum can be calculated from (7). It gave a current density of $18.3 \mathrm{~mA}$ and open circuit voltage of $1.1 \mathrm{~V}$. The efficiency thus obtained was $20 \%$.

4.3. Thermal Calculations. In the presence of QWs, the amount of heat dissipated in each region is given by (11):

$$
P_{H \lambda}=\sum_{k=0}\left(\Delta E_{e c k}+\Delta E_{h c k}\right) \phi_{0 k}\left(1-e^{-\alpha_{k \lambda} W_{k}}\right) \frac{W}{m^{2}},
$$




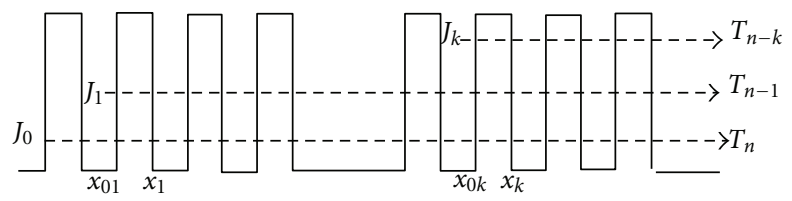

FIGURE 4: Tunneling through superlattice.

where

$$
\begin{aligned}
\Delta E_{\text {eck }}+\Delta E_{h c k} & =h v-\left(E_{g}+\Delta E_{c}+\Delta E_{v}\right), \\
\phi_{0 k} & =\phi_{0} \mathrm{e}^{-\sum_{m=1}^{k-1} \alpha_{m} W_{m}} .
\end{aligned}
$$

Here $\Delta E_{c}, \Delta E_{v}$ is the energy level below the electron energy in the conduction band and energy level below the hole energy in the valance band, respectively, $\alpha_{k}$-absorption coefficient of $k$ th region and $W_{k}$-thickness of the $k$ th region. Here, the high energy carriers settle on closest quantized energy level provided by the superlattice structure. Our modeling also took into account heat-up due to this small energy loss.

\section{Modeling and Results}

In our modeling, absorption coefficient is not a constant but a function of wavelengths for AM1.5 solar spectrum obtained from [10]. Superlattices in $\mathrm{p}$ and $\mathrm{i}$ regions were included to prevent scattering of high energy electrons. However, we did not consider energy scattering in contact areas. This is because they are very thin, and carriers will not have sufficient time to reduce their energies while traversing the contacts; thus recombination heating was not considered.

Our modeling assumed that QW energy levels in $\mathrm{p}$ and i regions were not aligned before illumination. Under solar radiation, the built-in potential step of $\mathrm{p}-\mathrm{i}-\mathrm{n}$ junction will be eliminated, thereby aligning the energy levels in $\mathrm{p}$ and $\mathrm{i}$ regions. This ensures carrier tunneling and transport across the junction.

Temperature increase for each wavelength of incident photon was calculated using the theory presented in Section 1 through 3 for cases with and without quantum wells, respectively. Figure 5(a) presents the distribution of temperature increase caused by photons of different wavelengths. It is clear that photons of energy about $2.5 \mathrm{eV}(490 \mathrm{~nm})$ bring about maximum temperature rise. The cumulative impact is presented by summation of all temperature rises. With no superlattices, the cumulative increase of temperature per unit of time is $8^{\circ} \mathrm{C} / \mathrm{s}$. As a result, the heating up by energetic carriers would attain a thermodynamic equilibrium at some temperature above the ambient temperature. However, the time to reach this equilibrium is not a concern of our modeling.

Designing superlattices in a, p, and i regions of our solar cell demonstrated that high energy carriers, having an option to occupy certain energy levels provided by our design, become less harmful as far as cell heating is considered. While analyzing the effect of quantum wells on temperature rise, it was assumed that the photons of energies lying

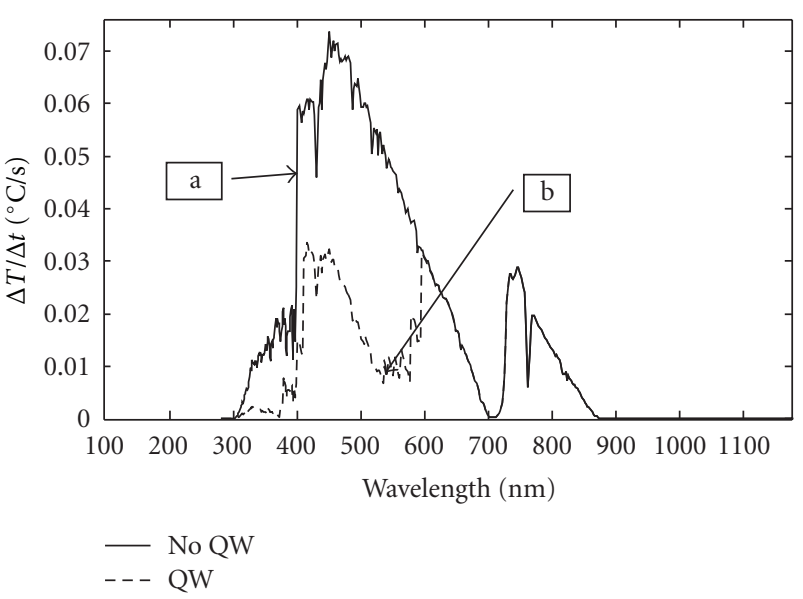

FIgure 5: Temperature distribution (a) without QW and (b) with QW.

within the gaps between the discrete energy levels would excite the charge carriers to energy levels just below such a gap. Figure 5(b) shows the temperature distribution when $\mathrm{p}$ and $\mathrm{i}$ regions are superlattice structures. The cumulative temperature rise is about $4^{\circ} \mathrm{C} / \mathrm{s}$.

Clearly, there is significant reduction in the temperature rise. The $10 \AA$ width barriers would ensure quantum tunneling of electrons through the barrier. The wider band gap also ensured that the barriers were optically inactive.

\section{Conclusion}

A formalized approach has been presented to assess the heating impact of high energy carriers. Modeling of current in superlattice structures for a solar cell under illumination was also proposed. Modeling shows that heat-up per unit of time was $8.5^{\circ} \mathrm{C} / \mathrm{s}$ with no quantum wells (cumulative) for a p-i-n heterostructure solar cell. As a remedy to solar cell heating, we designed superlattice structure with $30 \AA$ wide quantum wells and $10 \AA$ wide barriers in $\mathrm{p}$ and $\mathrm{i}$ regions. The modeling shows that the cumulative temperature rise per unit of time of operational solar cell equipped with superlattices was about $4^{\circ} \mathrm{C} / \mathrm{s}$. This implies a reduction of heating up per unit of time by a factor 2 . Modeling results also indicate reduction in current with the inclusion of quantum wells. This was expected as there would be no carrier generation inside barriers by photons with longer wavelengths. However, such a reduction can be remedied by increasing the thickness of the $\mathrm{p}$ and i regions. Our design ensures enhanced performance of a solar cell as reduction in heat-up leads to higher efficiency.

\section{References}

[1] J. J. Wysocki and P. Rappaport, "Effect of temperature on photovoltaic solar energy conversion," Journal of Applied Physics, vol. 31, no. 3, pp. 571-578, 1960.

[2] D. L. King, J. A. Kratochvil, and W. E. Boyson, "Temperature coefficients for PV modules and arrays: Measurement methods, difficulties, and results," in Proceedings of the IEEE 26th 
Photovoltaic Specialists Conference, pp. 1183-1186, October 1997.

[3] A. J. Nozik, "Spectroscopy and hot electron relaxation dynamics in semiconductor quantum wells and quantum dots," Annual Review of Physical Chemistry, vol. 52, pp. 193-231, 2001.

[4] M. F. Lamorte and D. H. Abbott, "Cascade solar cell design for high temperature operations," IEEE Transactions on Electron Devices, vol. 27, no. 4, pp. 831-840, 1980.

[5] S. Mil'shtein, A. Pillai, D. P. Nair, and A. Karumuri, "Heterostructure p-i-n solar cell with high efficiency," in Proceedings of the International Conference on Low Dimensional Structures \& Devices, 2011.

[6] E. Aperathitis, C. G. Scott, D. Sands, V. Foukaraki, Z. Hatzopoulos, and P. Panayotatos, "Effect of temperature on GaAs/ AlGaAs multiple quantum well solar cells," Materials Science and Engineering B, vol. 51, no. 1-3, pp. 85-89, 1998.

[7] S. M. Sze, Physics of Semiconductor Devices.

[8] J. Singh, Semiconductor Devices: An Introduction.

[9] R. Tsu and L. Esaki, "Tunneling in a finite superlattice," Applied Physics Letters, vol. 22, no. 11, pp. 562-564, 1973.

[10] http://refractiveindex.info/. 

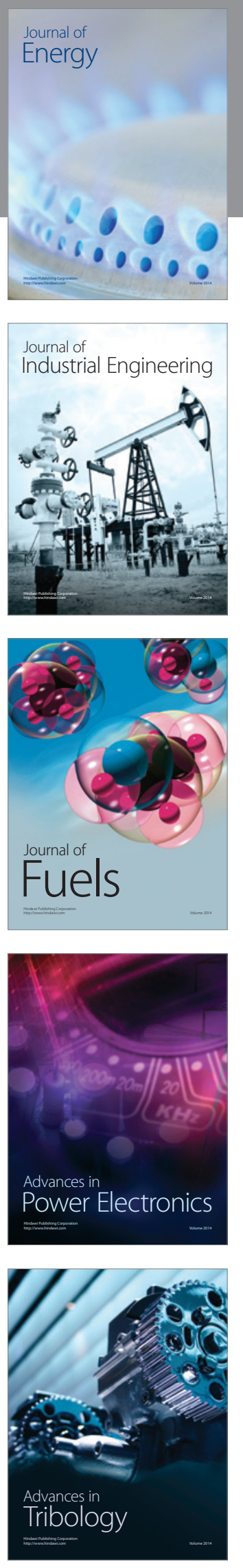
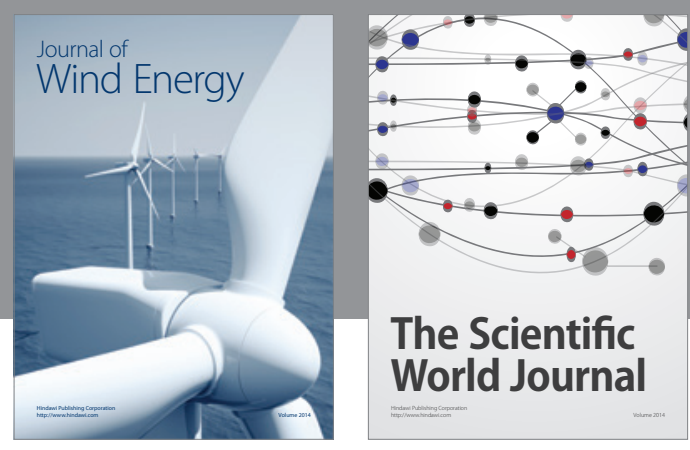

The Scientific World Journal

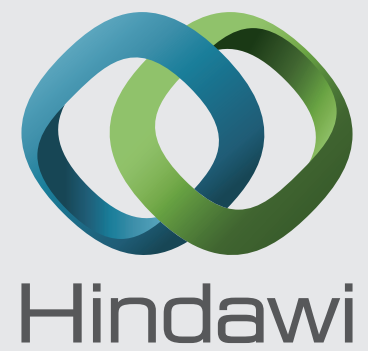

Submit your manuscripts at http://www.hindawi.com
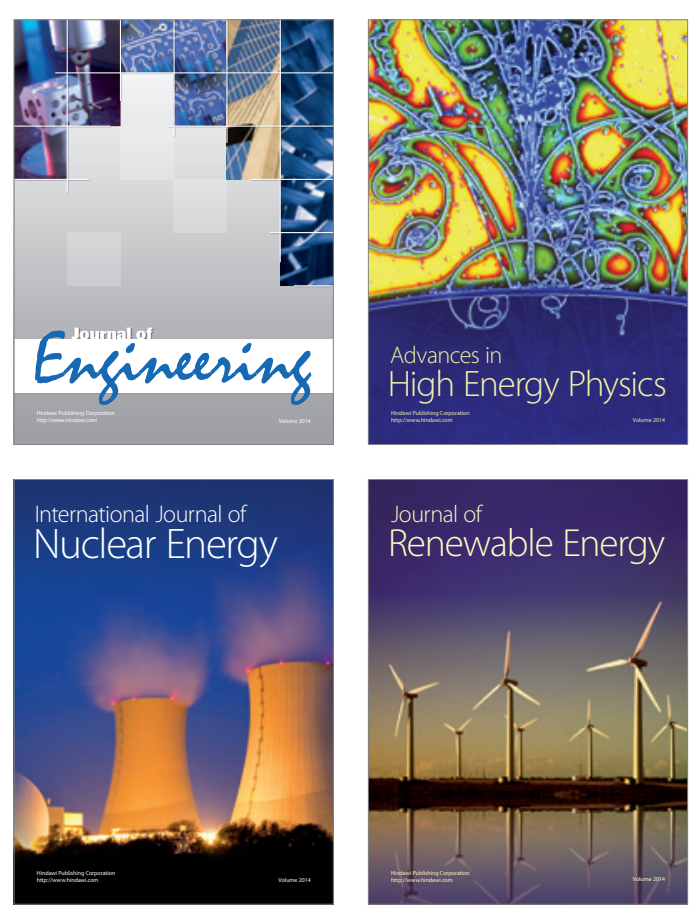

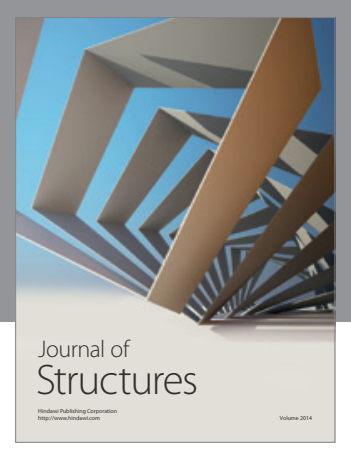

Rotating
Mechinery
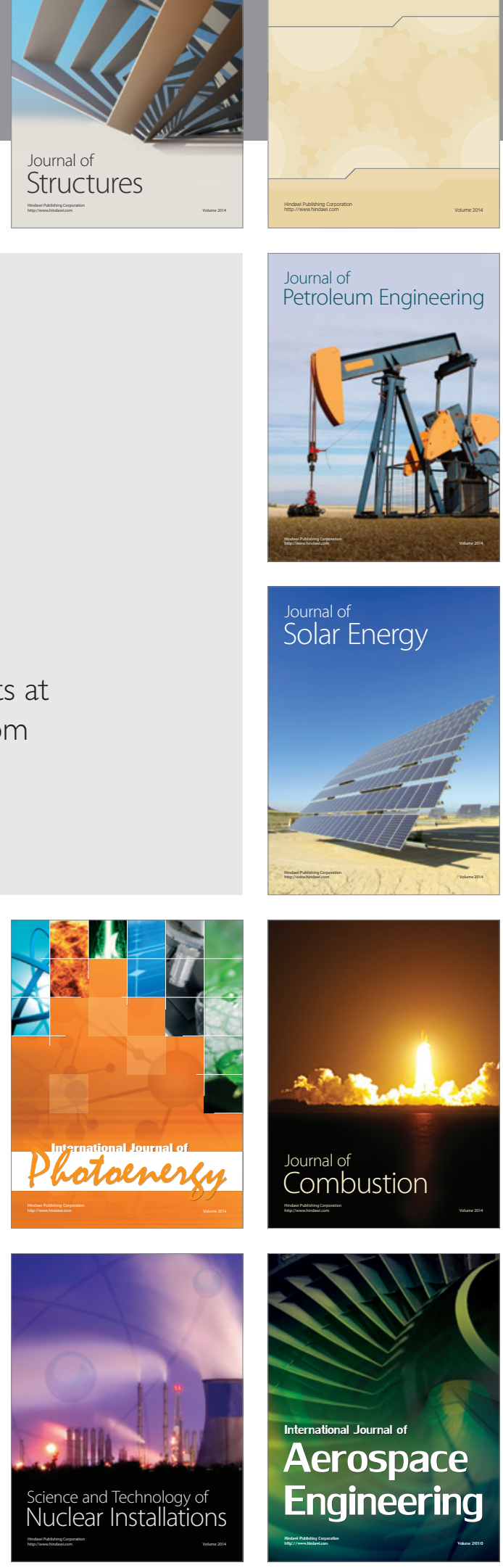